\title{
Jacinto Benavente en el modernismo hispánico: reflexiones publicadas en la prensa y amistades literarias
}

\author{
Jacinto Benavente's Stand on Spanish Modernism: \\ the Thoughts He Published in the Press \\ and his Literary Friendships
}

\author{
Diana Muela Bermejo \\ Universidad de Zaragoza \\ dmuela@unizar.es \\ ORCID iD: https://orcid.org/0000-0001-6162-6603
}

\begin{abstract}
RESUMEN
Desde sus primeras publicaciones en la prensa, Benavente trató de promover la visión artística del teatro a partir de distintas perspectivas: manifiestos, artículos de opinión, escenas breves, etc. Apoyó la creación y el desarrollo de revistas modernistas y participó en las que, en los años sucesivos, se convertirían en las más representativas del movimiento. Además, mantuvo una relación constante con los escritores modernistas de mayor reconocimiento: Valle-Inclán, Martínez Sierra, a quien apadrinó en sus inicios literarios y Rubén Darío, con quien se profesó siempre una profunda admiración. Este artículo contribuye a consolidar la situación de Benavente en el modernismo hispánico a través de estos aspectos.
\end{abstract}

Palabras Clave: Benavente; modernismo; teatro español; literatura y periodismo.

\begin{abstract}
From his earliest press publications, Benavente tried to promote the artistic vision of theatre from different perspectives: manifestoes, think pieces, short scenes etc. He supported the creation and development of modernist magazines, and he participated in those which, later on, would become the most representative of the movement. Besides, he kept a constant relationship with the most renowned modernist writers: Valle-Inclán, Martínez Sierra, whom he patronized at the beginning of his literary career, and Rubén Darío, whom he deeply admired. This article is a contribution to strengthen Benavente's position within Spanish Modernism through those aspects.
\end{abstract}

Key words: Benavente; Modernism; Spanish Drama; Literature and journalism. 
La relación literaria entre Jacinto Benavente y el modernismo ha sido analizada con cierta frecuencia en las últimas décadas del siglo XX debido, fundamentalmente, a la polémica existente en torno a la labor renovadora o tradicionalista del teatro benaventino y a la revalorización de los textos que engrosan el Teatro fantástico — vid., a este respecto, el estudio comparativo de Young (1970: 934-944) de Rosas de otoño, Primavera de otoño y Canción de otoño en primavera; el recorrido por los elementos simbólicos y fantásticos del teatro benaventino de González López (1977: 308-326) y, especialmente, la introducción al Teatro fantástico de Huerta Calvo y Peral Vega (2001: 9-85)-. Entre los estudiosos que han catalogado parte del primer teatro benaventino como modernista o estetista destacan Díaz-Plaja (1956: 63-64), Amorós (1992: 1601-1608), García Plata (en menor medida, no tanto como modernista sino como atraído por ciertos rasgos específicos del simbolismo, 2000: 89-109), Rubio Jiménez (2005b: 339-365) y Plata (desde el análisis de La gata de Angora, 2008: 95-123). Frente a éstos, el tradicionalismo de D. Jacinto fue apuntado por Fernández Martín (1982: 85-96) —en este caso ligado, especialmente, al teatro de Echegaray - y Salaün — que sólo considera a Benavente renovador en la ideología social (2000: 219-241) — y, a medio camino entre las nuevas estéticas finiseculares y la tradición realista anterior lo situó González López (1973: 454-465).

Es perceptible, a través de estos estudios, una clara evolución desde los años setenta hasta la fecha, en los que se ha producido un acercamiento a la visión de Benavente como renovador frente a los anteriores, probablemente condicionada por la edición de Teatro fantástico de Huerta Calvo y Peral Vega, publicada en 2001. El análisis de los escritos en la prensa iniciado por Rubio Jiméne $z^{1}$ ha abierto una vía necesaria para completar la relación entre el dramaturgo madrileño y los preceptos y escritores modernistas, que no debe ceñirse únicamente a sus obras representadas.

\section{ReFlexiones y MANifiestos de BenAVENTE SOBRE El CONCEPTO DE «MODERNISMO»: PRINCIPIOS GENERALES, LLAMAMIENTO \\ A LOS POETAS E IDEAS SOBRE EL TEATRO ARTÍSTICO}

La última década del siglo XIX es considerada el inicio de una nueva estética literaria y teatral en España, pues trasluce la voluntad de un grupo de jóvenes escritores de emprender un camino estético alejado de los modelos

\footnotetext{
${ }^{1}$ Rubio situó tres textos de Benavente («El teatro de los poetas», «Teatro artístico»y «Manifiesto del Teatro de Arte») en su edición de los textos que explicaban la renovación teatral española de 1900 (1998: 101-103; 213-218) y explicó en su introducción las líneas generales de los llamamientos a los poetas y del Teatro Artístico, que ya había tratado en su estudio de 1982.
} 
anteriores. Si bien todavía resulta una época temprana para el estreno de las que luego serán consideradas las más afamadas obras benaventinas (hasta $R o$ sas de otoño, en 1905, tampoco contará con el beneplácito pleno de público y crítica $^{2}$ ), comienzan a ver la luz en la prensa del momento una serie de artículos y escritos breves que suponen verdaderos manifiestos literarios de una nueva era de las letras españolas.

Benavente se refirió al modernismo en estos artículos tanto desde una perspectiva crítica como creadora, atendiendo, por una parte, a la recepción de la obra de otros autores (como sucedió tras la llegada de Darío a España o con la publicación de algunos textos de Valle-Inclán) y, por otra, llamando a los jóvenes poetas que buscaban en su lenguaje la belleza y el arte a publicar sus textos en las nuevas revistas.

El elemento común a ambas perspectivas es, indudablemente, el contacto que Benavente mantuvo con escritores de muy variados principios estéticos, englobados bajo el marbete «modernista» que, con sentido peyorativo, aglutinaba formas y contenidos muy dispares en un cajón de sastre de complicada delimitación. Estas relaciones, que serán analizadas con mayor detalle en apartados siguientes, le inspiraron la redacción de uno de los textos fundacionales del modernismo benaventino, que ya explicó Rubio Jiménez (1982b: 211)3: «Maestros de párvulos», publicado en el otoño de 1897 y en 1899 con el título «Teatro Artístico», como texto-programa del proyecto (vid. infra). En él, Benavente (1897a: 3) defendió el valor artístico del teatro y animó a los autores a educar al público y a alejarse del acomodamiento estructural en el que se hallaban sumidos desde hacía varias décadas.

Sin embargo, la fama que ha cobrado este artículo ha eclipsado otro de los textos fundamentales por su carácter temprano que relacionan a Benavente con el grupo de jóvenes modernistas que, en 1897, se hallaban en el máximo fervor defensor de esta estética. Se trata del prólogo que publicó a Mujeres de Fernández Vaamonde y que acompañaba a una presentación de Gaspar Núñez de Arce que, hasta la fecha, no ha sido analizado. En él se defendió de los reproches de un «crítico insigne» (probablemente Clarín) que «declaró desde su Vaticano, insignificantes o nulos a todos los escritores jóvenes de España» (1897c: 14) y apoyó al modernismo, al que atribuyó los siguientes principios estéticos: arte decadente por intelectual, pues se complace en sí mismo y nace y muere en él, no en el fin social; atribuido al artista moderno afirmado sólo en su egotismo y, por último, arte de aristocrática anarquía no sometido a ninguna estética ni a preceptos morales sino a la belleza (ibíd.: 14-16).

2 Vid., a este respecto, el capítulo primero de Muela Bermejo (2017) donde se desarrolla la recepción de la obra benaventina entre 1892 y 1905.

${ }^{3}$ Este texto se publicó a raíz del intento de Benavente de crear con el Teatro Artístico un teatro en España similar el Théâtre Libre de André Antoine, paralelo a los intentos de Adrià Gual en Cataluña (Rubio Jiménez 1982b: 211). 
Clarín (1897: 190) criticó a Benavente por haber publicado el prólogo de un libro que consideraba de poca calidad artística y de una estética a la que no respetó ni apoyó nunca. A pesar de la simpatía que el dramaturgo madrileño le generaba (aunque no lo conocía personalmente en aquellas fechas, habría que esperar un año después para que lo llevara a la dirección de la redacción de Madrid Cómico) le reprochó pertenecer a este nuevo movimiento, aunque le auguró éxito en el futuro por su carácter culto y europeísta. Ciertamente, Alas siempre ensalzó la pluma de Benavente, con la excepción de sus advertencias tras el prólogo citado. Elogió el trabajo de Figulinas (1898a: 3) y, cuando D. Jacinto asumió la jefatura de redacción de Madrid Cómico, escribió los siguientes versos:

El redactor en jefe, que actualmente

es mi amigo Jacinto Benavente:

erudito, cortés, sincero y franco,

hombre de mucho gusto; y yo me alegro

porque con asesor tan competente

cuando él diga que blanco, diré blanco

y cuando diga negro, diré negro (1898b: 5).

Criticó también positivamente el estreno de Cuento de amor (1899: 1) y apoyó la labor del Teatro Artístico y las propuestas de La gata de Angora, que habían sido cuestionadas tanto desde el punto de vista formal como por el contenido moral de la obra.

Además de «Maestros de párvulos»y del prólogo a Mujeres se mostró partidario del modernismo en otro artículo, publicado en 1898 como la tercera de sus «Cartas a Colombina» ${ }^{4}$ donde, por una parte, se declaró «esteta» y defensor del arte por el arte pero alejado de las modas bohemia y decadente y, por otra, se burló de la impostada admiración por D’Annunzio, que se había erigido como emblema de los incompris (1898a: 2).

No obstante, reflexionó de forma más seria sobre este concepto en «Modernismo. Nuevos moldes» en marzo de ese mismo año. Esta escena se recogería en la edición de Teatro fantástico de 1905, lo que constituye un hecho de enorme significación, pues no sólo da cuenta de la visión de D. Jacinto y, de la época en general, de lo que supuso la entrada del modernismo teatral a España sino, también, de que este teatro se ciñó fundamentalmente al terreno de la lectura, no al entorno de la representación. La etiqueta «nuevos moldes» procede de una discusión anterior que se inició hacia 1892 y remite a la relación

${ }^{4}$ González de Sande (2007) analizó la presencia de la commedia dell'arte en Los intereses creados desde el propio texto y con el apoyo de dos artículos, uno de ellos «Ibsen» (no en su primera versión). No obstante, creo que es necesario un recorrido previo por la commedia dell'arte desde Teatro fantástico a Los intereses creados desde el punto de vista de la creación, que debiera pasar por el tratamiento de Arlequín a Colombina en estas cartas. 
entre novela y teatro (Rubio Jiménez 1982b: 78). Galdós contribuyó en gran medida a esta discusión con un artículo que dedicó a la decadencia teatral, «Viejos y nuevos moldes», en 1893 y Clarín participó también de ella (Rubio Jiménez 1982b: 101 y Rubio Jiménez 1998: 84-90).

En este texto, Benavente censuró el papel de los críticos y el encasillamiento estético al que conducían las denominaciones genéricas, que impedían a los jóvenes escritores llevar a cabo la deseada renovación de la escena española. Para el dramaturgo madrileño, cuyo pensamiento queda reflejado en las palabras del Modernista, la situación española era una nueva «Querelle des Anciens et des Modernes», pues la rebeldía de los jóvenes contra los moldes establecidos había permanecido siempre viva en la historia de la literatura europea (1898d: 204-205).

Retomó el desagrado que le producía el significado de los términos «esteta», «modernista»y «decadente» en «Sermón perdido», pues no respondía a la actitud de los literatos jóvenes que, en toda Europa, promulgaban su interés por el arte verdadero (1898h: 1). Existía, para él, una confusión muy peligrosa para el desarrollo de la literatura entre el modernismo como gusto estético y como actitud vital, pues terminaba confundiéndose con la bohemia y con el rechazo a las normas de convivencia en sociedad, cuando debía ser concebido únicamente como principio de creación artística. Ideas similares desarrolló dos meses después en «Filosofía de la Historia» (1898c: 4).

Como parte de la generación de jóvenes artistas, Benavente apoyó a los pintores prerrafaelitas que fueron también criticados por «modernistas». Ellos promovían, como los poetas en el teatro, un acercamiento al arte primigenio, todavía no contaminado con los prejuicios de la sociedad (1898f: 8). En este sentido publicó, una década después, en junio de 1912, un artículo sobre el pintor Anselmo Miguel Nieto, con motivo de uno de sus retratos de Valle-Inclán que expuso en el salón de La Tribuna en 1912, junto a doce retratos femeninos, que le consagraron en Madrid (Serrano 2015: 266):

Es un retrato que siempre se asocia al arte renacentista [...] donde los críticos manejan un plantel de modelos diversos, en especial de la escuela veneciana y principalmente como paradigma la obra de Tiziano, aunque surgen también los nombres de Tintoretto y del Greco [...]. Este patrón, según varios de dichos autores, tiene su raíz en «la devoción de Valle al Tiziano» (Rivas Cherif) y, por lo tanto, Anselmo Miguel reflejó al escritor tal y como él quería verse en el lienzo (Serrano 2015: 272).

Benavente se hizo eco de esta captación por parte del pintor, pues lo elogió por ser capaz de ver más allá de la apariencia física (1912a: 5); en definitiva, por plasmar el modernismo (en la amplitud artística del término) de Valle-Inclán.

Por otra parte, el conservadurismo en las artes que reinaba en el grueso de la capital española encontraba en Cataluña su principal opositor, pues varios fueron los movimientos que trataron de acercarse al simbolismo teatral europeo 
y llevar el arte plástico a los escenarios (Rubio Jiménez 1982b: 44 y ss.). Benavente, en otro de sus manifiestos modernistas elogió a los artistas que lo llevaron a cabo en Sitges, centrándose especialmente en la figura de Rusiñol (1898e: 10). Meses después, a comienzos de 1899, será el propio Rusiñol el que diseñe el cartel para Interior de Maurice Maeterlinck, que habría de ser el que diera inicio al Teatro Artístico benaventino. Los meses de primavera a otoño de 1898 fueron, pues, especialmente fecundos en la publicación de artículos de opinión en los que Benavente ofreció su visión del modernismo teatral y artístico.

Los llamamientos de D. Jacinto al modernismo de los jóvenes escritores fraguaron en un proyecto que se llevó a cabo en los inicios de 1899: el Teatro Artístico, a pesar de que la idea se había forjado a finales del año anterior (Rubio Jiménez 2001: 42n). Al cartel diseñado por Rusiñol (vid. supra) lo acompañó su anuncio en La Vida Literaria, pero la representación no llegó a tener lugar. La primera se desarrolló en el Teatro de las Delicias de Carabanchel Alto - cuyo empresario era hijo de Antonio Vico- el 10 de septiembre de 1899, y fue la traducción de La fierecilla domada de Shakespeare, cuyo montaje fue dirigido, según expresó Ricardo Baroja, por Valle-Inclán (Martínez Sierra 2000: 216). Los ensayos habían comenzado el 25 de agosto en el salón de un palacio en la plaza Santa Ana y duraron dos semanas. Benavente representó el papel de Petruchio, Martínez Sierra el de Grumio, Pedro González Blanco el de Gremio y parece que la protagonista fue Concha Catalá. El estreno tuvo lugar a las ocho de la tarde y los carteles fueron nuevamente diseñados por Rusiñol, en la línea del de Interior de Maeterlinck. María Martínez Sierra ofreció sus recuerdos de esta representación en Gregorio y yo: medio siglo de colaboración (Martínez Sierra 2000: 98-99, 272).

Se representaron también en el marco del Teatro Artístico Cenizas de Valle-Inclán y Despedida cruel de Benavente, en el Teatro Romea tres meses después de la traducción shakespeariana, el 12 de diciembre de 1899. Conocido es el objetivo de la representación de Cenizas: reunir el dinero suficiente para comprarle un brazo ortopédico a Valle. Cuando la obra se publicó, Válle la dedicó a Benavente. Los ensayos tuvieron lugar en el estudio de Alejandro Saint-Aubin, y parece que también los dirigió el propio Valle-Inclán (Hormigón, 2006a: 274). Hormigón transcribe una carta de Valle a Martínez Sierra donde le pide que se incorpore al reparto de la obra, sustituyendo a Barinaga en el papel de Padre Rojas: "Querido Martínez Sierra: Le ruego que venga por el Café. Me parece que va usted a tener que hacer el papel de Jesuita en mi drama. Es favor que le agradecerá mucho su amigo Valle-Inclán» (2006b: 50). El estreno parece que fue retrasándose y la obra no convenció al público, especialmente por la interpretación de la actriz que daba vida a Octavia. Morano, Benavente y Martínez Sierra, por el contrario, recibieron críticas favorables (Fernández Almagro 1953: 69). Despedida cruel la representaron el propio Benavente, Josefina Blanco y, en el papel secundario, Gregorio Martínez Sierra, que apenas tenía frases. 
Sin embargo, el llamamiento a los poetas por parte de Benavente no concluyó en 1899 con el proyecto del Teatro Artístico, sino que continuó ocho años más tarde en su famoso artículo «El teatro de los poetas», en el que censuró la manida idea de la necesidad de romper moldes en el teatro, mientras que lo fundamental era para él «que alternen en el repertorio de las grandes compañías obras de los más diversos géneros y escuelas, para ampliar los gustos del público [...]» (1907b: 1). Las líneas siguientes resumen el nuevo llamamiento de Benavente a los artistas:

No sea sólo el Teatro la realidad y su prosa: vengan también la fantasía y los ensueños y hasta el delirar de la poesía. El Teatro necesita poetas [...] ¡Poetas de España, yo, que daría todas mis obras por un solo soneto de los vuestros, os lo digo con toda la verdad de mi amor a la poesía: venid al Teatro! (ibíd.: 1).

Un año después publicará Miquis el manifiesto del Teatro de Arte que firmará, entre muchos otros, Benavente, pues entre los años de 1907 a 1910 se estaba produciendo en España el debate sobre el teatro poético (Rubio Jiménez 2005a: 475-484). Benavente defendió en El Imparcial la primera representación del Teatro de Arte en el Teatro de la Ciudad Lineal en 1908, donde la obra elegida había sido El escultor de su alma de Ganivet que, «por ser tan poco de teatro - en el sentido profesional — era sin duda la más a propósito como obra de ensayo para el público y para los actores» (1908a: 3). Meses después apoyará a otro escritor modernista, Rodríguez Larreta, que había publicado una reconstrucción literaria del siglo XVII español: La gloria de don Ramiro (1908c: 4).

En este contexto Benavente escribió también El teatro del pueblo en 1909, no como llamamiento a los poetas sino como manifiesto de los ideales sobre los que debía elevarse una obra de teatro, apostando por las comedias y dramas en un acto - campo fecundo de tentativas escénicas - y defendiendo al público popular como «el único ante el que pueden representarse grandes obras, porque aunque no las entiendan, las intuyen» (1909b: 21). En esta cuestión difirió completamente de la opinión de Valle, que el 12 de diciembre de 1922 escribirá una carta a Cipriano de Rivas Cherif en términos opuestos:

\footnotetext{
El sentimiento de los espectadores crea la comedia, y aborta al autor dramático. ¿Quiénes son espectadores en las comedias? Padres honrados y tenderos, niñas idiotas, viejas con postizos, algún pollo majadero y un forastero. Los mismos que juegan la lotería en las tertulias de la clase media. Por eso los autores de comedias — desde Moratín hasta Benavente_- parecen nacidos bajo una mesa-camilla. Son fetos abortados en una tertulia casera. En sus comedias están todas las lágrimas de la baja y burguesa sensibilidad madrileña (Hormigón 2006b: 280).
}

Ese mismo año, además, Benavente había apoyado a algunos poetas —como Marquina - que, por la estética que perseguía en sus obras, había sido acusado 
de no poseer picardía teatral (1909a: 3) y fraguó también el proyecto del Teatro de los Niños, cuyo fundamento ideológico guardaba estrecha relación con el pensamiento expresado en El teatro del pueblo, con el objetivo de trasladar al teatro infantil la idea de teatro artístico. En la función inaugural participaron Darío y María Martínez Sierra, y poco después se representó una obra de Valle (vid infra) $)^{5}$.

En estos proyectos, Benavente trató de poner en práctica las ideas sobre el modernismo que llevaba expresando en la prensa más de una década pero no había logrado consolidar en Madrid. Además, en 1909 ya contaba con el apoyo mayoritario de público y crítica $\mathrm{y}$, por otra parte, el teatro poético encontraba un mayor número de adeptos entre los jóvenes dramaturgos. Benavente se había convertido, a través de estos textos y de sus relaciones personales, en precursor ideológico y, al mismo tiempo, maestro para ellos, pues contribuyó a impulsar un movimiento de difícil aceptación para el público adinerado y acomodado en sus gustos teatrales.

\section{Relación con los eSCRITORES MODERnistas: Darío, VAlle-INCláN y LOS MARTÍNEZ SIERRA}

A su labor de impulsor del modernismo llevada a cabo en la prensa madrileña se une la amistad que mantuvo con cuatro de los principales escritores modernistas del momento: Darío, Valle-Inclán y el matrimonio Martínez Sierra. De estas tres relaciones se conservan datos en las biografías de los respectivos autores, amén de algunas cartas y anécdotas recogidas en memorias. Recojo y completo, así, en los siguientes apartados los hechos conocidos pero tratados desde la perspectiva benaventina y añado otros que se deducen de la nueva documentación que hoy se posee.

\subsection{Ramón María del Valle-Inclán}

A finales de 1895 tuvo lugar el primer contacto entre Benavente y Valle-Inclán del que tenemos constancia. Ambos escritores acudieron a la tertulia de Ruiz Contreras, quien reunía en su estudio a multitud de jóvenes escritores (Hormigón, 2006: 186). Conocida es la labor que tuvieron las tertulias de salón y de café en el desarrollo de la literatura y las artes en estos años. En el caso del modernismo fue fundamental, pues contribuyó a la consolidación del sentimiento de rebeldía de los jóvenes estetas, que alimentaban y, sobre todo, creaban la moda a través de una nueva actitud vital y literaria. Conocido es, también, que Benavente y Valle se unieron en varias tertulias, en las que gene-

\footnotetext{
5 Vid. Huerta Calvo (2012) para un estudio detallado del Teatro de los Niños benaventino.
} 
ralmente se constituían como líderes. Sin embargo, en no pocas ocasiones se ha afirmado que la ruptura de la tertulia común supuso también el fin de su amistad, lo que se aleja completamente de la realidad, pues la admiración y el cariño mutuos fue constante hasta la muerte del escritor gallego, como tratan de demostrar las líneas que siguen.

La primera tertulia de café que frecuentaban ambos escritores fue la del Café Inglés de Madrid, considerada entonces el centro de la bohemia estética madrileña. Varios de los literatos y críticos que allí acudían habían asistido también a la de Ruiz Contreras (Bueno, Palomero, Dicenta, etc.). Hormigón recuperó un fragmento de $\mathrm{El}$ combate de Ricardo Castrovido donde se describe esta tertulia y se define a Benavente como una mordaz dama del siglo $\mathrm{XVIII}^{6}$, de gran ingenio y cultura literaria, «delicado, élite, parisiense» (2006: 192) en contraposición a otros escritores, como Dicenta o el propio Valle.

En estos meses de 1895 (que corresponden con los inicios literarios del dramaturgo madrileño, que tan sólo había publicado la primera versión del Teatro fantástico, Versos, Cartas de mujeres y estrenado El nido ajeno) Benavente y Valle guardaban una estrecha relación, que se traducía en grandes paseos nocturnos por las calles del Madrid antiguo, de lo que queda constancia en buena parte de los textos biográficos que se acercan a esta cuestión.

Poco después, sus nombres comenzaron a figurar conjuntamente en proyectos de volúmenes modernistas, como la «Colección flirt» de Ruiz Contreras, de la que sólo se publicó Epitalamio de Valle, a pesar de estar programados unos cuentos de Benavente que nunca vieron la luz 7 . El 15 de abril de 1897 Benavente redactó un breve apunte muy elogioso sobre Epitalamio, donde calificó al escritor gallego de «aristócrata del arte», igualándolo a Shelley, Rossetti, D’Annunzio, etc. (1897b: 81). Fue uno de los primeros en ponderar así la imagen de Valle-Inclán frente a la visión peyorativa de «esteta» que de él se tenía, y cuya caricatura pudo inspirar parte del Teófilo Everit de La comida de las fieras. En estas mismas fechas se afirma que ambos separaron sus tertulias: la de Benavente se marchó a la Cervecería Inglesa y la de Valle a la Horchatería Candelas (Lázaro 1930: 22), pero siguieron manteniendo su amistad:

Como si entre Valle-Inclán y yo hubiera habido alguna vez insidias y deslealtades. Nada más inexacto. En nuestra amistad no hubo mudanzas ni altibajos nunca. Por

${ }^{6}$ Precisamente serán jóvenes damas francesas del siglo XVIII las que protagonicen una de las obras de Benavente más ingeniosas y mordaces: Amor de amar, de 1902.

7 Tengo constancia, a través del legado que conserva el Archivo Histórico Nacional, de la existencia de algunos cuentos y leyendas escritos por D. Jacinto que corresponden a una etapa anterior a ésta. Sin embargo, no se conocen otros distintos a los que publicó en la prensa en los años siguientes, buena parte de ellos recogidos en Yagüe, 2014 y, otros, en los índices bibliográficos que conforman un anexo a mi tesis doctoral (Universidad de Zaragoza, 2015, inédita). Se desconoce, pues, cuáles fueron los previstos para la publicación de la colección de Ruiz Contreras. 
azares de nuestras vidas respectivas tal vez pasaba tiempo sin comunicarnos. Nuestra amistad no se enfrió nunca por eso, y siempre era una alegría para mí, y estoy seguro de que también para él, cuando volvíamos a encontrarnos (Benavente 1958: 123).

Esta amistad quedó reflejada en la composición y estreno de La comida de las fieras, que formó parte del intento frustrado de Valle-Inclán de iniciar una carrera actoral. La obra resulta de interés no sólo porque trasluce el de Benavente por traer el teatro francés coetáneo a los escenarios españoles sino, también, porque el personaje de Teófilo Everit, modernista, fue diseñado para Valle-Inclán ${ }^{8}$. No obstante, este personaje no corresponde únicamente con la exageración de algunos rasgos de Valle-Inclán sino que, sobre todo, escenifica el credo estético de los que se denominaban «estetas» o «decadentes» y del propio Benavente, que no era el mismo, aunque se les encasillara a todos bajo el denominador común de «modernistas». La idea de la defensa del arte y de la belleza, de la vuelta a los orígenes primigenios del teatro es común a ambos, pero la exageración de la actitud (que ejemplifica la quema del teatrillo de títeres o la descripción de la protagonista como «dama del renacimiento»); la mera pose en el comportamiento o la defensa vaga de una estética sin reflexión profunda es claramente parodiada por el dramaturgo madrileño. En definitiva, la experiencia de La comida de las fieras pone de manifiesto no sólo la amistad (y respeto mutuo) que había entre Benavente y Valle en 1898 sino, sobre todo, que ambos poseían la misma voluntad renovadora, con idénticos principios e intereses aunque, después, sus obras caminasen por senderos temáticos y formales distintos.

Tras el éxito de La comida de las fieras se dio en el Café de Fornos un banquete en honor a Benavente al que acudieron numerosas personalidades literarias, periodísticas y teatrales del momento, entre ellas, por supuesto, Valle-Inclán (M. B. 1898: 1).

A consecuencia de este estreno, de la forma compositiva de Gente conocida y de La farándula y de la novedad que ofrecían sus escenas publicadas en la prensa, todavía en 1900 se seguía tachando a Benavente de «modernista» con sentido peyorativo. Las críticas que sufrió tras el estreno de La gata de Angora llevaron a Valle-Inclán y a otros amigos a defender con fervor la modernidad del dramaturgo madrileño, de lo que da cuenta Almagro San Martín:

La Comedia llena, muchos críticos, ganas de meterse con D. Jacinto, como si la gente quisiera desquitarse de los aplausos que le otorgara, aunque con discrepancias, en Gente conocida y La comida de las fieras. Se nota en el aire verdadera «hincha» contra el modernismo, del que el vulgo considera a Jacinto como principal representante [...] Valle-Inclán, que estaba entre las butacas, se empeñó en

\footnotetext{
${ }^{8}$ Para los detalles de esta representación vid. Fernández Almagro (1953: 10), Hormigón (2006a: 245) y Rubio Jiménez (2001: 28).
} 
aplaudir contra viento y marea cuando las protestas contra la obra y Jacinto eran mayores. ¡Se armó la de San Quintín! Hubo bofetadas y bastonazos. A Valle se lo llevaron detenido (Almagro San Martín 2013: 332-333).

La separación de las tertulias no condicionó, en absoluto, su amistad - como prueban estas líneas y, sobre todo, el desarrollo del proyecto del Teatro Artístico (vid. supra) — pues ésta no se fundamentaba en un aprecio personal sino en un enorme respeto intelectual, que trascendió también las posiciones opuestas que mantuvieron durante la Gran Guerra.

A partir de los primeros años del siglo XX continuó la amistad entre los dos escritores, pero tanto su participación en proyectos comunes como las anécdotas que otros coetáneos recogieron van adquiriendo, conforme avanzan los años, un carácter más ocasional ${ }^{9}$.

Desde 1902 a 1905 no consta nada que uniera a Benavente y a Valle más allá de su coincidencia en algún café, su colaboración en revistas modernistas o de afán renovador ${ }^{10}$ y su participación en homenajes. Precisamente el 4 de noviembre de 1904 se celebró en el Café Inglés un banquete homenaje a Valle-Inclán, al que asistió D. Jacinto (el Café Inglés albergaba cierto significado en la unión de los dos escritores, pues fue la primera tertulia de café a la que acudieron conjuntamente durante algún tiempo).

Poco después, en los albores de 1905 se produjo un distanciamiento ideológico (no personal) entre los dos escritores, que actuaron de forma opuesta en la firma del conocido manifiesto contra concesión del Premio Nobel a Echegaray. Según Almagro San Martín, suponía la defensa de Benavente como su dramaturgo, consagrando así su nombre en el panorama teatral frente al ya consolidado Echegaray (Montero Alonso 1967: 162-167). Sin embargo, no creo que la firma del documento significase un espaldarazo para Benavente, sino que fue más bien un manifiesto de credo u oposición estética a las formas teatrales neorrománticas que entraban en contradicción con el afán renovador de la «gente nueva». Reducir el objetivo del manifiesto a la figura de Benavente es, en mi opinión, ofrecer una visión sesgada de los principios literarios que defendían los escritores de la azoriniana Generación del 98. Por otra parte, el hecho de que D. Jacinto no lo firmara no suscitó la censura por parte de sus coetáneos, sino que éstos más bien respetaron la actitud del dramaturgo aunque no la compartieran y lo siguieron considerando como uno de los grandes reno-

91899 fue, además, un año fundamental en la producción periodística de Benavente. A su paso como director (durante cuatro meses) de Madrid Cómico le siguió la creación y desarrollo de La Vida Literaria, revista que apoyó los nuevos nombres modernistas y publicó textos de los escritores consolidados. Para un estudio detallado del paso de Benavente por Madrid Cómico y de las características y evolución de La Vida Literaria remito a Versteeg (2009: 425-438) y Muela Bermejo (2014: 309-326) respectivamente.

${ }_{10}$ Para la participación de Benavente en Helios, vid. el apartado dedicado a los Martínez Sierra. 
vadores de las letras y la escena españolas, a pesar de que este hecho haya sido juzgado en más de una ocasión como el desencadenante del alejamiento de D. Jacinto de sus coetáneos. En realidad, Benavente nunca estuvo demasiado ligado a otros escritores, mantuvo siempre su independencia, desde sus inicios literarios hasta bien entrada su madurez artística.

Tras la firma del manifiesto contra Echegaray comenzó una serie de artículos en los que Benavente escribió acerca del concepto de «regeneracionismo» en literatura, en los que tuvo un protagonismo especial la figura de Valle-Inclán. En El Liberal comparó su obra con otros libros publicados que él denominó «de la derrota», por centrarse en las pérdidas españolas y en la «monomanía regeneradora» (1907a: 1). Este texto sí supuso un claro alejamiento de las ideas de algunos de sus coetáneos:

Hay una nueva hornada de escritores jóvenes — de esos que se cartean con don Miguel de Unamuno en grandes pliegos de papel comercial, cartas que serán algún día como Evangelios. ¡Y trabajo le mando al que pretenda concordarlas!(1907a: 1).

La crítica era, ante todo, un manifiesto modernista, motivo por el cual ensalzó la obra de Valle, lo que justifica la diferenciación, al menos en la época, entre modernistas y regeneracionistas: «Bellos de su propia belleza, libros que no parecen de ahora, entre toda esa literatura de castigo que pretende hacernos a todos mejores, y sólo ella, con lamentable modestia, se exceptúa, de modo que es peor cada día» (1907a: 1). El 31 de mayo de 1909 volvió a tratar este asunto al comentar El resplandor en la hoguera de D. Ramón pero, a diferencia del anterior, lo insertó dentro del marco de la literatura política y de guerras civiles, donde los autores siempre terminaban tomando partido por uno u otro bando y trasladándolo a los textos; a pesar de lo cual elogió el trabajo de Valle. Al respecto de este artículo explica Hormigón:

El 2 de junio, Joaquín Argamasilla de la Cerda publica en El Correo Español una Carta abierta a D. Jacinto Benavente, en la que intenta responder a las opiniones vertidas por éste en su artículo del día 31 de mayo: «Usted, una gloria española, cree que los carlistas guerrearon por cosas que el día antes no les importaban, y que no debieron importarles nunca. No he de intentar yo, insigne señor mío, convencerle de lo contrario, pues dada la marcial idiotez que usted de modo implícito, pero generoso, nos atribuye a todos, temo que ni siquiera escuchara mis argumentos. Por otra parte, en lo referente a los carlistas, el prodigioso artista que es Valle-Inclán espero que logrará, cuando avance en la publicación de sus episodios, reivindicarnos ante usted del inconsciente espíritu que nos supone» (2006a: 474).

Las críticas de Benavente, sin embargo, no iban dirigidas tanto a la ideología carlista como a la creación de obras con fines políticos mermando su interés artístico. Por ello, continuó su apoyo al teatro de Valle-Inclán en el 
marco del Teatro de los Niños un año después, pues incluyó en su repertorio La cabeza de dragón, que se estrenó con éxito en la Comedia el 5 de marzo de 1910 (Hormigón 2006a: 501). Unos meses después, en julio de 1910, durante su estancia en Argentina, el escritor gallego dio una conferencia sobre el modernismo, donde habló de pintura y literatura y destacó a Azorín, Unamuno y Benavente: «los únicos escritores de libros que aquí son completamente conocidos y reconocidos» (Hormigón 2006b: 132). Así pues, Valle-Inclán está presente en todos los proyectos renovadores del teatro de Benavente de forma continuada y, recíprocamente, D. Jacinto lo está en las reflexiones de Valle sobre el modernismo. Sin embargo, cuando se produjo poco después el conflicto de El embrujado, Valle pidió a Benavente que presidiera su lectura pública - hasta fue a visitarle para concretar algunos aspectos de la misma- (Hormigón 2006a: 632), pero este finalmente no lo hizo, ocupando su lugar Pérez Díaz (Valle-Inclán 1994: 13). Ambos escritores serán nombrados en noviembre de ese año miembros de la ejecutiva de la Academia de la Poesía, que integraban también Martínez Sierra, Villaespesa y Rodríguez Marín.

A partir del año 1914, la mayoría de textos que relacionan a Benavente y a Valle-Inclán corresponden a entrevistas o conferencias impartidas por éste en las que mencionó a D. Jacinto o analizó su obra, amén de los artículos y cartas que surgieron en torno a los manifiestos aliadófilo y germanófilos, en los que ambos escritores respetaron sus opuestas posiciones ${ }^{11}$.

Una excepción a la polémica que suscitó la Gran Guerra fue el elogio benaventino de Voces de gesta, fechado el 27 de mayo de 1912, donde ensalzó la obra de Valle por haber sido publicada antes que representada (1912b: 3). Un año antes ya había apuntado esta idea con motivo de la reseña de Ícara de Sellés:

11 Vid., a este respecto, Fernández Almagro (1953: 177) donde explica brevemente el manifiesto firmado por los aliadófilos; Hormigón (2006a: 688-689), donde da noticia de unas declaraciones de Benavente a Avelló en las que el dramaturgo madrileño se manifestó germanófilo y defendió la neutralidad de España; Hormigón, 2006b: 170-173 donde se transcriben unas cartas de Valle al director de La Correspondencia de España publicadas en este periódico en respuesta a las ideas de Benavente manifestadas en El Correo Español y Valle-Inclán (1994: 157), la carta que envió Valle al periódico. Es, a su vez, la trascripción de unas líneas de la entrevista que Manuel Avelló celebró con Benavente, «Una visita extraña»»». Un extranjero pretendía sondear a D. Jacinto sobre su opinión acerca de los franceses y éste respondió con las razones de su animadversión hacia el pueblo galo: en numerosas ocasiones habían manifestado que no eran amigos de los españoles y, sobre todo, durante la cuestión marroquí. Benavente se declaró neutral ante la posible llegada de una guerra y explicó que él no alentaba a los escritores a animar a España para que entrara a favor de los aliados porque sus simpatías estaban del lado de Alemania. Valle-Inclán respetó siempre la opinión de Benavente: «Me parece acertada la opinión de Benavente - comienza diciéndonos el gran don Ramón-. Creo que es la única postura lícita para un hombre que, aceptando el hecho, la realidad de su existencia y reconociendo las virtudes y defectos del fascismo y del comunismo, permanece equidistante de las dos tendencias» (Valle-Inclán 1994: 604). 
Lo cierto es que, sin contar las que han dejado de escribirse, en la seguridad de que no hubieran sido admitidas por ninguna empresa, acaso las mejores obras dramáticas de estos últimos años impresas andan sin haber logrado el favor de ese amplio criterio. Díganlo las tragedias bárbaras de Valle-Inclán Águila de blasón y Romance de lobos [...] (1911a: 3).

A partir de este momento, apenas se conservan algunas palabras sueltas que dirigió Valle a la obra de Benavente en algún artículo o entrevista, de carácter deslavazado y sin un hilo crítico común. El más relevante, por lo contradictorio con la opinión de la época, data de 1914 y fue recuperado por Joaquín y Javier del Valle-Inclán con el título «Volvamos al teatro. Valle-Inclán y La Malquerida. Don Benito Pérez Galdós. Un poco de estética». En él, el escritor gallego negó a La Malquerida la condición de mejor obra benaventina a pesar de su carga emocional intachable, pues consideraba mejor El dragón de fuego, «obra magistral e inmensa» (Valle-Inclán, 1994: 137). Su estreno, sin embargo, obtuvo una pésima recepción y ninguno de los grandes críticos del momento destacó esta obra por encima de Los intereses creados, La noche del sábado, Rosas de otoño o La malquerida. Sin embargo, el carácter poético de la pieza, amén de su enorme carga visual, justifican la admiración que D. Ramón sentía por ella, pues priorizaba el interés artístico por encima de la representabilidad. Por ello, muchos años después Valle afirmó que los textos de Benavente no podían ser transpuestos al cinematógrafo, pues se dirigían al sentido estético del oído y no de la vista (Valle-Inclán, 1994: 129); a pesar de lo cual reconoció que, de sus contemporáneos, era el único cuyo teatro leía - aun cuando no se representara - dado el interés artístico de sus diálogos (Valle-Inclán, 1994: 403).

Valle-Inclán siempre consideró a Benavente un maestro dialoguista, además del mejor dramaturgo del momento. Ensalzó la forma de sus dramas y comedias pero también su contenido, situándolo en el Parnaso de autores teatrales españoles:

Jacinto Benavente podrá estrenar una obra que no obtenga éxito: pero la suma de sus obras es extraordinaria, es de una totalidad tan considerable como la de Tirso de Molina o Lope de Vega. Ha tratado todos los asuntos y todos los ambientes con un concepto de universalidad que le coloca en el propio rango de nuestros autores clásicos (Valle-Inclán 1994: 416).

Además de las entrevistas o conferencias en las que Valle habló sobre Benavente, se constatan desde los años diez del siglo XX algunas apariciones conjuntas (en persona o pluma) en determinados actos o acontecimientos artísticos, de los que da cuenta Hormigón (2006a: 348, 594 y 596, 651, 696-697). El 6 de diciembre de 1930 se celebró un homenaje a Jacinto Benavente y a María Palou en Madrid, en el que el escritor gallego leyó unas palabras que resumen la amistad, personal y literaria, entre ambos: 
Al cabo de los años no he dejado de ser su amigo y soy su admirador. Creo que ha puesto en pie a todo un teatro. Porque no se es autor dramático por el hecho de haber escrito cuatro o seis bellas comedias, no; se es completo autor dramático cuando se crea todo un escenario. Así, Lope de Vega. Y así, Jacinto Benavente (Valle-Inclán 1994: 443-444).

La amistad se vio truncada por la muerte de Valle-Inclán el 5 de enero de 1936 pero, nueve años después de su fallecimiento, D. Jacinto mostró reciprocidad al expresar su admiración por él prologando sus Obras completas a petición de Josefina Blanco:

Mi querida amiga: [...] nada tiene que agradecerme; soy yo el agradecido y satisfecho. Al releer ahora las obras en la edición completa una vez más he gozado en su lectura y una vez más he comprendido la grandeza del escritor único en la literatura española. Cuando se lee lo que ahora se escribe, lo que ahora pasa por bueno, se siente uno más orgulloso de haber sido contemporáneo de Valle-Inclán, de haberle conocido en todas sus genialidades, y en toda su grandeza (Valle-Inclán 2008: 277).

Ambos mantuvieron una breve correspondencia y, finalmente, aunque con algo de retraso ${ }^{12}$, apareció el prólogo prometido en 1944, que reproducía con modificaciones la misiva original (Rubio Jiménez 2001: 208). En este prólogo Benavente trazó un retrato literario a través de sus recuerdos de Valle desde su primer encuentro, a finales del siglo XIX, a la llegada de este a Madrid. Rememoró los inicios de su amistad en largos paseos nocturnos por la capital y sus discusiones sobre diferentes artes, contrarias a la mayoría de críticos y tertulianos y en las que ellos solían estar de acuerdo (1958: 119). En esencia, Benavente trató en este prólogo de modificar la idea generalizada de la personalidad de Valle: subrayó su timidez, su carácter prudente y amable con las personas con las que trabó verdadero afecto y su cabal pensamiento literario y artístico. La faceta polemista del escritor gallego pasó a un segundo plano, convirtiéndolo en un Cyrano al que muy pocas personas llegaron a conocer (ibídem). Su admiración traspasaba el plano personal y consideraba a Valle el escritor más completo de su generación, no sólo novelista sino dramaturgo y poeta (ibíd.: 125).

Así pues, la amistad entre Benavente y Valle, que duró casi cuarenta años, se mantuvo constante más allá del plano literario, en el que los dos escritores comenzaron a distanciarse pasados los albores del modernismo. Ambos siempre recordaron su labor de renovación de las letras y estimaron las virtudes formales de sus obras y, a pesar de la opinión de muchos críticos, respetaron siempre su posición ideológica, que nunca llegó a distanciarles. El modernismo de Benavente está íntimamente relacionado con la figura de Valle-Inclán con el

${ }^{12}$ Escribe D. Jacinto a Josefina: «Mi querida amiga: el prólogo está terminado. Hubiera querido enviarlo con los Reyes Magos pero no he tenido tiempo de hacerlo copiar y corregirlo. Se lo enviaré lo más pronto posible, el 15 lo más tarde» (Valle-Inclán 1994: 278). 
que, a pesar de las enormes diferencias existentes entre sus obras, siempre compartió el mismo espíritu de culto a la belleza y al arte.

\subsection{Rubén Dario}

Muy distinta a la de Valle-Inclán (y mucho menos documentada que ésta) fue la relación que Benavente mantuvo con Rubén Darío tras la llegada de este a España en diciembre de 1898. Al instalarse en Madrid, comenzaron a trabar amistad a través, sobre todo, de las tertulias (Darío, 1917a: 169). El 19 de noviembre, un mes antes de su llegada, Benavente le había dedicado un elogioso artículo donde reprochaba a los críticos españoles que juzgaban las obras de los americanos como si de escritores peninsulares se tratara y calificaban a Darío de «poco castizo» (Benavente 1898g: 11). El nicaragüense representaba para él el exponente máximo de habla hispana y lo igualó a los simbolistas franceses (ibídem), como diez años antes hiciera Valera en su carta a Darío con motivo de la publicación de Azul... De todos sus textos, el preferido por Benavente era Prosas profanas y otros poemas, que se había publicado dos años antes de la aparición de este artículo.

La relación entre Rubén Darío y Jacinto Benavente se mantuvo, en estos meses, cercana y afectiva; ambos intercambiaron varios textos dedicados a la reflexión sobre la obra del otro y la admiración que se profesaron late en las palabras de aliento y apoyo mutuo que se dirigieron.

El 3 de marzo de 1899, al poco tiempo de su estancia en la capital española, Darío escribió uno de los textos más importantes que publicó sobre Jacinto Benavente y otros escritores del momento: «La joven literatura», que vio la luz después en España Contemporánea. Resulta curioso que, ese mismo día, el nicaragüense le dedicara también un poema, «Los retratos», que publicó en la revista Juan Rana (1899: 2). Para Darío,

Jacinto Benavente es aquel que sonríe. Dicen que es mefistofélico, y bien pudieran ocultarse entre sus finas botas de mundano dos patas de chivo. Es el que sonríe: ¡temible! Se teme su crítica florentina más que los pesados mandobles de los magulladores diplomados; fino y cruel, ha llegado a ser en poco tiempo príncipe de su península artística, indudablemente exótica en la literatura del garbanzo. Se ha dedicado especialmente al teatro, y ha impuesto su lección objetiva de belleza a la generalidad desconcertada (1907: 86).

La mayoría de retratos literarios de Benavente en la época se centran sobre todo en su carácter irónico y en su crítica mordaz, pero éste añade algo más: el perfil de Benavente como cultivador del arte y la belleza frente al realismo y su singular personalidad, aislada del común de las letras españolas del momento.

Darío vio también, en la obra de Benavente, la influencia directa de algunos escritores franceses - Gyp, Lavedan y Prévost, principalmente-. Des- 
tacó Teatro fantástico, cuyos textos consideraba joyas por tratarse de un «teatro en libertad» comparable a los diálogos shakespearianos. Parece, según consta en este texto, que Benavente le había encargado una obra para que se representara en el proyecto de teatro libre que hacía tiempo trataba de llevar a cabo, aunque Darío dudaba de su éxito por los gustos alejados del público (1907: 86).

Así pues, la relación entre Darío y Benavente fue, sobre todo, literaria, de respeto y admiración mutuos; y hasta el momento no se conservan más datos que algunos artículos y anécdotas biográficas que prueben que dicha amistad fuera más allá del terreno de las letras. El valor que otorgaba D. Jacinto a Darío como poeta le llevó a criticar su nombramiento como embajador, pues como gran poeta debía, en su opinión, dedicarse exclusivamente a la poesía (1908b: 4). A pesar de ello, se relacionó con la figura diplomática de Darío, pues contribuyó a la creación de un álbum con las firmas de varios escritores renombrados - Pardo Bazán, Valle, Galdós, etc. - que iba a ser entregado al Ministro de Relaciones Exteriores de Nicaragua con motivo de su matrimonio (Hormigón 2006a: 479).

De forma también oficial, Benavente presidió una fiesta celebrada en honor a Darío en el Ateneo de Madrid en 1912, que llevaba planeándose hacía tiempo pero las cortas estancias del nicaragüense en Madrid no lo habían permitido. Enrique Amado leyó el juicio crítico de Valera sobre Azul... y Andrés González-Blanco dio a conocer unos fragmentos del volumen que dedicó al poeta. La velada terminó con unas palabras de Barbadillo y una lectura de versos de Darío. Estaba previsto que Valle contribuyera con una disertación, pero no pudo hacerlo al verse obligado a emprender un viaje (Anónimo, 1912: 263-268). En su revista reprodujeron las palabras que D. Jacinto brindó a los asistentes, que consistieron en una loa a Darío y una irónica expresión de alegría por no tratarse de una fiesta necrológica ${ }^{13}$. Este año, además, Darío había publicado la sección «Cabezas», en la que dedicó otro retrato a Benavente y reflexionó sobre su entrada en la Academia ${ }^{14}$ con el objetivo, según él, de oficializar su triunfo ya logrado entre las masas populares (Darío 1917b: 4). Explicó, además, el teatro benaventino en relación con el mundo del boulevard y la vida parisiense pero con el ingenio y los diálogos propios de las comedias clásicas de Lope o Calderón modernizadas.

${ }^{13}$ Ese año, además, había pronunciado Benavente un discurso necrológico en el Ateneo al poeta Juan Maragall, que había fallecido el 20 de diciembre de 1911.

${ }_{14}$ Benavente nunca tomó posesión de su sillón académico. A este respecto afirmó Valle-Inclán en una entrevista publicada en La Esfera el 5 de marzo de 1927: «Hay un tipo de escritor que nunca será académico: Unamuno, Baroja, Blasco Ibáñez; yo desde luego... Este tipo de escritor no será académico, en primer término, porque no lo busca. Luego porque la Academia, con su espíritu, con sus normas, con su vida quieta, ata, apaga en el escritor lo que en él haya de independencia, de rebeldía, de libertad. Yo creo que Benavente se resiste por esto a leer su discurso de entrada...» (Valle-Inclán 1994: 339). 
No se conservan más datos que unan a los dos escritores hasta la muerte de Darío en 1916. Su fallecimiento terminó con una relación literaria, en torno al concepto de modernismo, que se había mantenido fiel a la admiración y respeto mutuos desde su comienzo en 1898; pero que no fue más allá del terreno de las letras $\mathrm{y}$, en menor medida, del oficial cuando así lo requirieron las circunstancias.

\subsection{Gregorio y María Martínez Sierra}

En los primeros meses de 1898 se inició la relación entre Jacinto Benavente y el joven matrimonio Martínez Sierra, a quienes el dramaturgo madrileño comenzó apadrinando en sus primeros pasos como escritores modernistas, a pesar de que María no llegó a conversar con él hasta 1911, tras el estreno de Canción de cuna (Martínez Sierra 2000: 93-94). El recuerdo que María Lejárraga tenía de Benavente no responde tanto a una amistad fraternal $-\mathrm{o}$, al menos, coetánea - como la que, por edad, mantenían Benavente y Valle, sino más bien a una ligazón paterno filial, de mecenazgo y apoyo (ibídem). La voluntad del dramaturgo madrileño de crear escuela modernista y la necesidad del matrimonio de despegar en el panorama de las letras españolas dieron lugar al desarrollo profesional de uno de los directores de escena más importantes en la historia del teatro español del siglo XX, especialmente por la labor de renovación emprendida con el Teatro de Arte ${ }^{15}$.

Este entusiasmo por el teatro artístico fue probablemente lo que llamó la atención de Benavente quien, a finales del siglo XIX, buscaba ese perfil en los jóvenes escritores. En este sentido, Martínez Sierra pudo verse influido en su Teatro de ensueño por el Teatro fantástico benaventino (de hecho, Pastoral está dedicada al dramaturgo madrileño); pero, sobre todo, por su común afición a Shakespeare, de la que ya había dejado constancia María en Gregorio y yo (Martínez Sierra 2000: 98) ${ }^{16}$.

El 24 de diciembre de 1898 Benavente publicó en Madrid Cómico su prólogo al libro de Martínez Sierra El poema del trabajo. Se trata de un prólogo poético, metáfora del proceso de escritura, donde Benavente le elogió como escritor de sentimientos más que de palabras, que rendía culto a la belleza, paralelamente a lo escrito con anterioridad sobre Darío. En la nota que precede al prólogo, Benavente calificó a Martínez Sierra de «joven equilibrado» (1898b: 12) en el que no había «alardes de psicología decadente ni alambicados

15 Young explica en términos generales la relación entre Benavente y los Martínez Sierra en la línea del paternalismo protector benaventino, aunque con ciertos matices diferenciadores: para Martínez Sierra el estilo y los personajes son los aspectos dominantes, mientras que para Benavente lo son las ideas y la construcción del drama en torno a ellas (Young 1970: 935).

16 Salaün observa una especial influencia de Benavente en Saltimbanquis de Martínez Sierra. 
refinamientos de poesía artificial»(1898b: 12). Le sorprendía que estuviera escrito en prosa, pues en sus inicios casi todos los poetas jóvenes tendían a la expresión poética, que terminaba convirtiéndose en repetición de tópicos o en mera adición de metáforas sin sentido. Benavente defendió, así, la consolidación del poema en prosa modernista en España.

Este prólogo supuso para los Martínez Sierra un gran apoyo en su lanzamiento como escritores, tanto es así que María cuenta en Gregorio y yo que, a fuerza de leerlo, lo aprendió de memoria (Martínez Sierra 2000: 95). En la primavera de 1899 se publicaron los Diálogos fantásticos de Martínez Sierra en El Álbum de Madrid, con una dedicatoria a Jacinto Benavente (quien publicó allí algunas de sus Cartas de mujeres), por haber sido impulsor y maestro de los jóvenes modernistas.

Muy poco después de los estrenos del Teatro Artístico (en los que participó Gregorio como actor en las dos obras citadas anteriormente: la adaptación de La fierecilla domada y Despedida cruel, aunque con papeles menores) tuvo lugar lo que María Martínez Sierra consideraba el espaldarazo definitivo de Benavente a la obra de ambos (Martínez Sierra 2000: 95). En el número almanaque de 1900 de la revista Instantáneas Benavente publicó un texto con el título «Filosofías», que en realidad había escrito Gregorio. Manuel Salvi, editor de la revista, pedía a escritores consagrados que publicaran textos suyos cuyo asunto girara en torno a las fotografías que en ella se publicaban y a Benavente le pidió que compusiera uno sobre un tren, que él aceptó pero con cierta contrariedad por la nimiedad del asunto. Por ello, pidió a Gregorio que lo escribiera por él, quien aceptó gustoso sintiéndose honrado por el maestro y, así, el texto se publicó con la firma de D. Jacinto.

A partir de este momento, lo que unió a Benavente con los Martínez Sierra fue su participación en revistas modernistas y, con menor importancia, la celebración de homenajes o eventos en torno a diversas figuras del arte. Una de ellas es la revista Helios, que recogió textos de Benavente y Valle bajo la coordinación de Juan Ramón Jiménez y un grupo de jóvenes escritores, entre otros, Martínez Sierra. Benavente publicó en Helios varios textos, pero sus colaboraciones fueron siempre obras breves o fragmentos de dramas más extensos que se habían estrenado previamente, muy distintas al concepto de escena suelta que manejaba en sus contribuciones a revistas de la época en los años anteriores.

En el primer número de la revista se publicó el cuadro $\mathrm{V}$ de La noche del sábado con la presentación «Novela escénica, dividida en cinco cuadros». La denominación de «novela escénica» aporta el sentido de la publicación de este texto, cuya composición se aleja de la división tradicional en actos y de la delimitación histórica de géneros y se acerca a las novedades teatrales que ofrecía el teatro francés coetáneo ${ }^{17}$. En la confusión terminológica entre «inno-

17 Para un análisis detallado de La noche del sábado vid. mi estudio de 2017. 
vador», «renovador» y «modernista», tal y como lo entendemos hoy, reside la razón de la configuración de la personalidad de Benavente como «modernista» o «esteta» en aquellos años, cuyo teatro camina por senderos muy distintos a los de Valle o Martínez Sierra, cuya catalogación es similar. El objetivo común de cambiar las letras españolas y su filiación a diversos manifiestos y proyectos a favor del arte en el teatro conlleva la inmediata etiquetación de todos ellos como «modernistas», englobando después técnicas y formas dramáticas muy dispares.

El número siguiente se inicia con un texto de Pedro González Blanco sobre Benavente, retratando al dramaturgo madrileño como:

El más feliz de los innovadores en el teatro español contemporáneo [...] Benavente marca un punto de partida en la literatura dramática, buscando tan sólo la vida, la vida que quiere extinguirse, la vida privada, reveladora de las mentiras que por todas partes nos rodean (González Blanco 1903: 129-130).

Defiende, además, el concepto de «escena» que en España desarrolló D. Jacinto:

El teatro, se decía antes, es la representación de acciones y de grandes ideas que se adoptan. La vida es una sucesión de actos, de pequeñas acciones, muchas veces poco dramáticas, pero que determinan crisis violentas en los individuos. Es infinitamente más artístico hacer interesantes los hechos simples que tienen por móviles inmensos sufrimientos, que trazar a grandes rasgos la historia de acciones casi gigantescas llevadas a cabo por personajes casi inverosímiles (ibíd.: 131).

Este texto se convierte en un verdadero ensayo defensor del modernismo benaventino y trata, esencialmente, de compararlo con las técnicas tradicionales de composición dramática y de creación de personajes.

En el número octavo de Helios se publicó la comedia completa Por qué se $a m a$, que había estrenado recientemente la compañía Guerrero-Mendoza y, en el número noveno, apareció Los favoritos, basada en un episodio del Much Ado About Nothing de Shakespeare. Hasta el año siguiente no se volverá a publicar otra obra de Benavente, en este caso se tratará del drama breve La casa de la dicha, que había estrenado en el Teatro de las Artes de Barcelona la noche del 9 de diciembre de 1903 la compañía del Teatre Íntim.

$\mathrm{Su}$ dramaturgia renovadora, la participación en revistas modernistas, su impulso del teatro artístico en España y el padrinazgo de algunos jóvenes poetas valieron a Benavente el homenaje literario que varios escritores le rindieron en la revista Renacimiento, que dirigía Gregorio Martínez Sierra; cuatro años después de la última participación benaventina en Helios, ya en 1907. Rodríguez Moranta (2012: 26) explica cómo se construyó la revista bajo el respeto a la gente vieja, a través de la publicación de una serie de monográficos dedicados a los autores consagrados en el panorama nacional contemporáneo y se 
acerca a la amistad entre los Martínez Sierra y Benavente centrándose, sobre todo, en los números de Renacimiento y en otros datos biográficos que aquí se recogen (2012-2013: 241-246).

Así, en el primer número de Renacimiento se incluyó un apartado dedicado a Benavente, con opiniones sobre su persona y su obra de José Francés, Martínez Ruiz, Darío, Pardo Bazán y Martínez Sierra. La más extensa es la de Francés, quien subrayó el carácter poético y modernista de Benavente, presente especialmente en la trilogía La noche del sábado, El dragón de fuego y La princesa Bebé (1907: 105). Por su parte, Martínez Ruiz — que firmó su texto diez años antes - retomó en su retrato literario de Benavente la caracterización del dramaturgo madrileño como sátiro mordaz y mefistofélico pero verdadero artista por su cultura y análisis de la sociedad (1907: 113-114), aunque varias disputas los alejaran definitivamente tiempo después. De Emilia Pardo Bazán recogieron una crítica sobre la adaptación benaventina de Twelfth Night (que se había estrenado el 11 de marzo de 1899), publicada el 3 de abril de 1899 en La Ilustración Artística. En ella defendía la obra, que consideraba un cuento poético (1907: 114).

Martínez Sierra publicó un texto que había aparecido en su libro Motivos de 1905, donde reseñaba la edición de las Obras completas de Benavente en trece tomos (diez de ellos dedicados al teatro). Ensalzó, sin reparos, la labor dramatúrgica de su maestro con las siguientes atribuciones: sinceridad en las palabras, intelecto bien educado, inteligente análisis de la sociedad de su tiempo y voluntad para combatir los prejuicios a los que estaba sometida con una gran belleza en las formas (1907: 116). Por último, en el caso de Rubén Darío se transcribió de nuevo un fragmento de las líneas dedicadas a Teatro fantástico en «La joven literatura».

En el número siguiente de Renacimiento la figura de D. Jacinto continuó presente, en este caso a través de la publicación de La princesa sin corazón ${ }^{18}$. La revista dedicó en el número cuarto un homenaje a Darío similar al de Benavente, siguiendo el esquema habitual de publicación, en el que incluyeron la reproducción del texto del dramaturgo madrileño que había visto la luz en Madrid Cómico el 19 de noviembre de 1898 (vid. supra).

Martínez Sierra en 1907 ya no dependía literariamente de Benavente como en 1899; su espíritu inquieto y emprendedor le había llevado no sólo a la dirección de esta revista sino, también, a convertirse en nuevo anfitrión de la tertulia de Villaespesa, a la que se incorporaron, entre otros, Benavente y Valle. Parece que en este espacio, poco tiempo después, Valle y Martínez Sierra discutieron y dejaron de hablarse (Hormigón 2006a: 432).

18 Rodríguez Moranta (2012-2013: 250-251) recoge, además del análisis de los citados números de Renacimiento, una carta de Benavente a Martínez Sierra donde el dramaturgo madrileño le anuncia el envío de un fragmento de La princesa sin corazón. 
La amistad entre Martínez Sierra y Benavente, sin embargo, continuó como hasta el momento y en julio de ese año el dramaturgo madrileño reseñó Horas de sol en la revista La Lectura (1907c: 294-295). Prueba de esta continuidad es el hecho de que, cuatro años después, se produjera la primera visita de D. Jacinto a casa de los Martínez Sierra, tras el estreno de Canción de cuna (Teatro Lara, 21 de febrero de 1911). La anécdota es recordada por María y las visitas posteriores se hicieron muy frecuentes (Martínez Sierra 2000: 100). Además, Benavente reseñó Canción de cuna en El Imparcial en términos muy elogiosos:

Es una de las más bellas comedias estrenadas en los últimos años. Fui de los primeros en conocerla y en predecir su triunfo [...] Además... ¡ jesperaba desde hace tanto tiempo esta comedia! ¿Os acordáis, mi querido Gregorio, de aquella redacción del Madrid Cómico, y de aquel vuestro primer libro, y de vuestra timidez, que es el pudor de las inteligencias honradas; timidez y pudor que hoy desconocen tantos jovenzuelos osados que aún no escribieron una línea y ya creen haber conquistado el mundo? (1911b: 3).

Parece que Gregorio había dudado de la amistad de Benavente al no haber visto estrenada ninguna obra suya con anterioridad, opinión que se vio transformada a raíz de esta reseña. Poco después se dio un banquete en honor a Martínez Sierra para celebrar los éxitos de Canción de cuna y Primavera en otoño. Marquina leyó unos versos y la mesa presidencial la ocuparon Benavente, los Álvarez Quintero, Dicenta, Linares Rivas, Ramos Carrión, Marquina, Cándido Lara y Eduardo Yáñez. Entre los asistentes figuran Bernardo Candamo, Vicente Lleó, Catarineu, Amadeo Vives, Pérez de Ayala, Enrique Amado, Antonio Paso, García Álvarez, Thuillier, Felipe Trigo, etc.; quienes, al final del banquete, acordaron solicitar la concesión de la cruz de Alfonso XII a Martínez Sierra. En Diario Universal se reproduce el brindis de Marquina y el discurso de Gregorio, en el que coloca como estandarte del artista a la belleza, de la que aquél fue siempre siervo y agradeció especialmente a Benavente, Rusiñol y los Álvarez Quintero su ayuda y apoyo brindados desde sus inicios como escritor. De D. Jacinto constata su acogida bondadosa cuando tenía diecisiete años al prologarle su primer libro, Atrio:

Oyéndole hablar, se me descubrieron las leyes fundamentales del Teatro moderno, y aproveché sus lecciones sin descanso, porque he cumplido ayer los treinta años, pero a los diez y ocho ya escribía comedias. Esto puede explicarles a ustedes la coincidencia del dominio con la improvisación, que hace constar, en son de elogio un crítico, mi amigo (Anónimo 1911: 3).

Ese mismo año, además, Martínez Sierra publicó una introducción a Teatro de Benavente que vio la luz en París y en cuyo volumen se antologaron Rosas de otoño, Los intereses creados y Al natural. Para Gregorio, las dos condiciones fundamentales de D. Jacinto como dramaturgo eran el amor al arte y, sobre 
todo, la independencia y la inteligencia intuitiva (Martínez Sierra 1911: 4-10). En opinión de Salaün (2000: 53), este prólogo supone la filiación clara de Benavente como modernista a los ojos de Martínez Sierra.

Tras esta década desaparecen los testimonios que relacionan a Benavente con los jóvenes modernistas, en un momento en que se encontraba planteando otras posibilidades dramatúrgicas más ligadas a la comedia burguesa y al drama rural (La malquerida se entrenó, no en vano, a finales de 1913 y Señora ama — su comedia predilecta — había visto la luz en los escenarios en 1908). Los últimos llamamientos al teatro de los poetas y el proyecto del Teatro Artístico cierran un capítulo en la trayectoria literaria de D. Jacinto que sólo se verá completado, desde un punto de vista social, con diversas apariciones públicas o reseñas de apoyo a otros escritores modernistas.

En consecuencia, el modernismo de Jacinto Benavente debe ser analizado no sólo a través de sus estrenos y otras publicaciones literarias - como Teatro fantástico - sino, también, desde una perspectiva teórica y social que considere los manifiestos publicados en la prensa y sus amistades con otros escritores modernistas, que precisan de ciertas puntualizaciones. Resulta fundamental el estudio de los textos críticos que publicó desde los años noventa del siglo XIX porque son, por una parte, muestra de cómo se introdujo y consolidó el modernismo en España y, por otra, porque sitúan el papel que desempeñó el dramaturgo madrileño como uno de los líderes de la joven generación renovadora que profesaba un entusiasmo por el arte y la belleza trasladado a la poesía $\mathrm{y}$ a los escenarios.

Estos textos, de la mano de los testimonios sobre sus relaciones personales o profesionales, no traslucen una imagen completamente nueva del escritor ni distinta de la que hoy se conoce, sino que ordenan y sitúan a Jacinto Benavente dentro de un panorama modernista de difícil determinación, que debe ser completado con el análisis exhaustivo de sus dramas.

\section{FUENTES}

Almagro San Martín, Melchor (2013). Biografia de 1900, ed. de Amelina Correa Ramón. Granada: Universidad de Granada.

Anónimo (1911). «Banquete a Martínez Sierra», Diario Universal. 2951 (9/III), p. 3.

Anónimo (1912). «Fiesta de Rubén Darío en el Ateneo. Palabras de Benavente», Ateneo. XIII (enero-junio), pp. 263-268.

Benavente, Jacinto (1897a). «Maestros de párvulos», El Proscenio. 1 (26/IX), p. 3.

Benavente, Jacinto (1897b). «Notas de un lector», Revista Contemporánea. CVI (15/IV), p. 81. Benavente, Jacinto (1897c). «Prólogo», en Emilio Fernández Vaamonde, Mujeres. Madrid: Fernando Fe, p. 14.

Benavente, Jacinto (1898a). «Cartas a Colombina», Juan Rana. 14 (27/I), p. 2.

Benavente, Jacinto (1898b). «El poema del trabajo», Madrid Cómico. 827 (24/XII), p. 12.

Benavente, Jacinto (1898c). «Filosofía de la Historia», Madrid Cómico. 812 (10/IX), p. 4. 
Benavente, Jacinto (1898d). «Modernismo. Nuevos moldes», Madrid Cómico. 785 (5/III), pp. 204-205.

Benavente, Jacinto (1898e). «Notas de Arte», Madrid Cómico. 813 (17/IX), p. 10.

Benavente, Jacinto (1898f). «Notas sueltas», Madrid Cómico. 803 (9/VII), p. 8.

Benavente, Jacinto (1898g). «Rubén Darío», Madrid Cómico. 822 (19/XI), p. 11.

Benavente, Jacinto (1898h). «Sermón perdido», La Correspondencia de España. 14631 (14/ IV), p. 1.

Benavente, Jacinto (1903). «La noche del sábado. Acto V», Helios. 1 (IV), pp. 21-34.

Benavente, Jacinto (1907a). «Crónica. Libros gratos», El Liberal. 10180 (17/VII), p. 1.

Benavente, Jacinto (1907b). «El teatro de los poetas», Heraldo de Madrid. 6072 (13/VII), p. 1.

Benavente, Jacinto (1907c). «Horas de sol, de Martínez Sierra», La Lectura, año I, tomo 2, julio de 1907, pp. 294-295.

Benavente, Jacinto (1908a). «De sobremesa», El Imparcial. 14733 (1/VI), p. 3.

Benavente, Jacinto (1908b). «De sobremesa», El Imparcial. 14749 (8/VI), p. 4.

Benavente, Jacinto (1908c). «De sobremesa», El Imparcial. 14999 (14/XII), p. 4.

Benavente, Jacinto (1909a). «De sobremesa», El Imparcial. 15354 (6/XII), p. 3.

Benavente, Jacinto (1909b). El teatro del pueblo. Madrid: Fernando Fe.

Benavente, Jacinto (1911a). «De sobremesa», El Imparcial, año XLV, núm. 15820, 20/ III/1911, p. 3.

Benavente, Jacinto (1911b). «De sobremesa», El Imparcial. 15799 (27/II), p. 3.

Benavente, Jacinto (1912a). «Acotaciones», Nuevo Mundo. 963 (20/VI), p. 5.

Benavente, Jacinto (1912b). «De sobremesa», El Imparcial, año XLVI, núm. 16249, 27/V/1912, p. 3.

Benavente, Jacinto (1958). «Prólogo» a las Obras completas de Valle-Inclán, en Jacinto Benavente. Obras completas XI. Madrid: Aguilar, p. 123.

Clarín (1897). «Palique». Madrid Cómico. 746 (5/VI), p. 190.

Clarín (1898a). «Palique». Madrid Cómico. 799 (11/VI), p. 3.

Clarín (1898b). «Palique». Madrid Cómico. 805 (23/VII), p. 5.

Clarín (1899). «Revista mínima». La Publicidad. 7353 (28/III), p. 1.

Darío, Rubén (1899). «Los retratos», Juan Rana. 1 (3/III), p. 2.

Darío, Rubén (1907). «La joven literatura», en España Contemporánea. París: Garnier, 1907, p. 86.

Darío, Rubén (1917a). Autobiografía, en Obras completas. Vol. XV. Madrid: Mundo Latino, p. 169.

Darío, Rubén (1917b). Cabezas. Pensadores y artistas. Políticos. En Obras completas. Vol. XXII. Madrid: Mundo Latino.

Díaz-Plaja, Guillermo (1956). «Benavente y el Modernismo», Clavileño. 7, pp. 63-64.

Francés, José (1907). «Jacinto Benavente», Renacimiento. 1 (marzo), p. 105.

González Blanco, Pedro (1903). «Jacinto Benavente», Helios. 2 (V), pp. 129-130.

M. B., «Banquete a Benavente», El Globo. 8887 (14/XI), p. 1.

Martínez Ruiz, José (1907). «Opiniones», Renacimiento. 1 (marzo), pp. 113-114.

Martínez Sierra, Gregorio (1907). «Opiniones», Renacimiento. 1 (marzo), p. 116.

Martínez Sierra, Gregorio (1911). «Introducción» en Jacinto Benavente, Teatro. París: Thomas Nelson and Sons, pp. 4-10.

Pardo Bazán, Emilia (1907). «Opiniones», Renacimiento. 1 (marzo), p. 114.

Valle-Inclán, Joaquín del (2008). Valle-Inclán inédito.Madrid: Espasa-Calpe.

Valle-Inclán, Ramón María del (1994). Entrevistas, conferencias y cartas. ed. de Joaquín y Javier del Valle-Inclán. Valencia: Pre-textos. 


\section{BIBLIOGRAFÍA CITADA}

Amorós Guardiola, Andrés (1992). «Benavente y el teatro modernista», en Actas del X Congreso de la Asociación Internacional de Hispanistas. Barcelona: PPU, vol. 2, pp. 1601-1608.

Fernández Almagro, Melchor (1953). Vida y literatura de Valle-Inclán. Madrid: Editora Nacional.

Fernández Martín, María Isabel (1982). «Sobre la originalidad de los primeros dramas benaventinos», Anuario de Estudios Filológicos. 5, pp. 85-96.

García Plata, Valentina (2000). «Teatro fantástico de Jacinto Benavente: ¿una renovación dramatúrgica y escénica?», Siglo Diecinueve. 6, pp. 89-109.

González de Sande E. (2007). «La commedia dell'arte: fuente literaria del teatro español del siglo XX», Archivum: Revista de la Facultad de Filología. 57, pp. 69-90.

González López, Emilio (1973). «Benavente, punto y contrapunto de la generación del 98», Cuadernos Hispanoamericanos. 279, pp. 454-465.

González López, Emilio (1977). «El teatro de fantasía de Benavente», Cuadernos Hispanoamericanos. 320-321, pp. 308-326.

Hormigón, Juan Antonio (2006a). Valle-Inclán: biografía cronológica y epistolario. Volumen I: biografía cronológica (1866-1919). Madrid: Asociación de Directores de Escena de España.

Hormigón, Juan Antonio (2006b). Valle-Inclán: biografía cronológica y epistolario. Volumen III: epistolario. Madrid: Asociación de Directores de Escena de España.

Huerta Calvo, Javier (2012). «El Teatro de los Niños, de Jacinto Benavente», Don Galán. 2. Accesible en <http://teatro.es/contenidos/donGalan/donGalanNum2/pagina.php?$\mathrm{vol}=2 \& \mathrm{doc}=2 \quad 1>$

Huerta Calvo, Javier y Emilio Peral Vega (2001). «Introducción», en Teatro fantástico. Madrid: Espasa Calpe, pp. 9-85.

Lázaro, Ángel (1930). Biografía de Jacinto Benavente. Madrid: Compañía Iberoamericana de Publicaciones.

Martínez Sierra, María (2000). Gregorio y yo. Medio siglo de colaboración. Valencia: Pre-Textos.

Montero Alonso, José (1967). Jacinto Benavente: su vida y su teatro. Madrid: Sucesores de Rivadeneyra.

Muela Bermejo, Diana (2017). Hacia la renovación del teatro en los albores del siglo XX: Gente conocida, La noche del sábado y Rosas de otoño de Jacinto Benavente. Vigo: Academia del Hispanismo.

Muela Bermejo, Diana (2014). «La Vida Literaria (1899) como reflejo de una nueva estética finisecular», Anales de Literatura Española. 16, pp. 309-326.

Plata, Francisco (2008). «En busca del ideal: el arte y el artista en La gata de Angora, de Jacinto Benavente», Hecho Teatral. 8, pp. 95-123.

Rodríguez Moranta, Inmaculada (2012). La revista Renacimiento (1907). Una contribución al programa ético y estético del Modernismo hispánico. Vigo: Academia del Hispanismo.

Rodríguez Moranta, Inmaculada (2012-2013). «El teatro necesita poetas. Jacinto Benavente, maestro de Martínez Sierra», Journal of Hispanic Modernism. 12-13, pp. 241-250.

Rubio Jiménez, Jesús (1982a). «Colaboraciones de Benavente en la prensa madrileña: 18901900», Cuadernos Bibliográficos. 44, pp. 135-151.

Rubio Jiménez, Jesús (1982b). Ideología y teatro en España: 1890-1900. Zaragoza: Departamento de Filología Española. Universidad de Zaragoza-Libros Pórtico. 
Rubio Jiménez, Jesús (1998). La renovación teatral española de 1900. Madrid: Publicaciones de la Asociación de Directores de Escena de España.

Rubio Jiménez, Jesús (2001). Ramón del Valle-Inclán y Josefina Blanco: el pedestal de los sueños. Zaragoza: Prensas Universitarias de Zaragoza.

Rubio Jiménez, Jesús (2005a). «Eduardo Marquina y el Modernismo castizo en el teatro», Anales de Literatura Española Contemporánea. 30, pp. 457-484.

Rubio Jiménez, Jesús (2005b). «La poética teatral de Jacinto Benavente», en Mariano de Paco y Francisco Javier Díez de Revenga (coords.), Jacinto Benavente en el teatro español. Murcia: Fundación Cajamurcia, pp. 339-365.

Salaün, Serge (2000). «¡Ay, papá, cómo entras en el siglo XX! (Benavente o la ilusión de un teatro moderno)», Siglo Diecinueve. 6, pp. 219-241.

Serrano Alonso J. (2015). «El pintor de cámara de Valle-Inclán: Anselmo Miguel Nieto», Anales de Literatura Española Contemporánea. 40 (3), 263-302.

Vesteeg, Margot (2009). «Jacinto Benavente, director de Madrid Cómico», en Amparo de Juan y Javier Serrano (coords.), Literatura hispánica y prensa periódica (1875-1931): actas del congreso internacional. Santiago de Compostela: Universidad de Santiago, pp. 425-438.

Yagüe Ferrer, María Isabel (2014). Jacinto Benavente. Bibliografía general. Zaragoza: Prensas de la Universidad de Zaragoza.

Young, Raymond A. (1970). «Benavente, Martínez Sierra y Rubén Darío: una comparación», en Actas del Tercer Congreso Internacional de Hispanistas. México: Asociación Internacional de Hispanistas - El Colegio de México, pp. 935-944.

Fecha de recepción: 8 de julio de 2016 .

Fecha de aceptación: 25 de noviembre de 2016. 